

\title{
Functional and metabolic characteristics of peripheral blood mononuclear phagocytes in patients with different clinical courses of multiple sclerosis
}

\author{
O. M. Koliada*, N. I. Vdovichenko**, T. I. Kolyada**, O. P. Bilozorov** \\ *Kharkiv National Medical University, Kharkiv, Ukraine \\ **Mechnikov Institute of Microbiology and Immunology, Kharkiv, Ukraine
}

Article info

Received 07.10.2020

Received in revised form 14.11.2020

Accepted 15.11.2020

Kharkiv National Medical University of the Ministry of Health of Ukraine, Nauki ave., 4 ,

Kharkiv, 61022, Ukraine.

Tel.: +38-057-707-73-40

E-mail:

Pathophys.khnmu@gmail.com

Mechnikov Institute

of Microbiology and

Immunology of the National

Academy of Medical Science

of Ukraine, st. Pushkinska,

14-16, Kharkiv,

61057, Ukraine

Tel.: +38-057-731-31-51

E-mail:

imiamn@amnu.gov.ua

Koliada, O. M., Vdovichenko, N. I., Kolyada, T. I., \& Bilozorov, O. P. (2020). Functional and metabolic characteristics of peripheral blood mononuclear phagocytes in patients with different clinical courses of multiple sclerosis. Regulatory Mechanisms in Biosystems, 11(4), 494-500. doi:10.15421/022075

Functional and metabolic features of intact and stimulated mononuclear phagocytes were studied in patients with different clinical courses of multiple sclerosis, the study included 66 patients with relapsing-remitting and 32 patients with progressive course of multiple sclerosis. The state of the mononuclear phagocytes was characterized by expression of costimulatory molecules and direction of L-arginine metabolism. Relative quantities of CD80, CD86 and PD-L1 positive monocytes were determined with Phycoerytrin-labeled monoclonal antibodies in immunofluorescence test in peripheral blood and after culture in parallel series with addition of: (a) E.coli lipopolysaccharide (a stimulator of TLR4), (b) a single-stranded RNA - preparation ssRNA40/LyoVec (a stimulator of TLR7/8), (c) IL-4 (an anti-inflammatory interleukin). The formation of NO was determined by the amount of nitrite in the culture supernatants, arginase activity was determined in cell lysates of the monocyte fraction. We showed that functional and phenotypic characteristics of monocytes depend on the clinical course of multiple sclerosis. In patients with progressive course, the relative number of CD86+ cells was significantly higher and PD-L1+ cells significantly lower than in patients with relapsing-remitting course and healthy persons, in patients with relapsing-remitting course the number of PD-L1+ cells was increased. The number of CD80+ cells did not show any significant difference in the investigated groups of patients relative to the control group. In vitro stimulation of peripheral blood monocytes with TLR4/8 produced a significant increase in the number of CD86 + and decrease in the number of PD-L1+ cells in patients with the progressive course. In patients with the relapsing-remitting course LPS produced an increase in number of PD-L1+ cells. We did not find any difference in activity of the arginase pathway of L-arginine metabolism in the intact monocyte fraction of peripheral blood in patients with multiple sclerosis versus the control group, but stimulation with TLR4 agonist of mononuclear cells of patients with progressive course caused significant increased arginase activity versus baseline. At the same time, versus control cells arginase activity in patients with the progressive course decreased after LPS treatment, but trended to increase after TLR7/8 treatment. In patients with the relapsing-remitting course these changes had a similar direction but were less expressed. The results may be considered as an indication of the activation of peripheral blood monocytes and their polarization trend in the M1 direction in patients with the progressive course of multiple sclerosis, these changes could be considered as signs of violation of autoimmune regulatory mechanisms in multiple sclerosis.

Keywords: demyelinating disease; expression of cosignal molecules; L-arginine metabolism; stimulation of Toll-like receptors.

Introduction

The results of numerous studies indicate that in multiple sclerosis (MS) mononuclear phagocytes of the brain (microglia), as well as of peripheral organs and blood (monocytes, macrophages, dendritic cells) are a key pathogenetic element of initiation and maintenance of inflammation in the CNS (Goodin, 2014). It is known that mononuclear phagocytes are involved in various pathological processes at almost all stages of the disease. Thus, along with the activation of microglia cells, the most important mechanism for initiating and maintaining inflammation in the CNS is the infiltration of peripheral monocytes into brain tissue with their subsequent transformation into activated macrophages and dendritic cells. Peripheral monocytes are also involved in the formation of the pro-inflammatory context on both sides of the blood-brain barrier (BBB), of antigen presentation, activation and differentiation of lymphocytes, promote the penetration of activated lymphocytes through the BBB and have a wide spectrum of regulatory function (Goodin, 2014; Baufeld et al., 2018; Mammana et al., 2018). Activation of microglia can have both positive and harmful effects on the course of inflammation in the brain. Microglia cells as well as other mononuclear phagocytes exhibit significant phenotypic and functional heterogeneity and plasticity, an inflammatory microenvironment often leads to M1-type polarization of microglia, with the production of neurotoxic factors (e.g., eicosanoids, proinflammatory cytokines, complement components, proteinases (MMP), glutamate, NO, ROS), which leads to cell damage and neuronal death. In other conditions microglia can exhibit M2 phenotype properties and play an anti-inflammatory role by secreting neurotrophic growth factors and anti-inflammatory cytokines. Neurological damage itself can contribute to excessive activation of microglia, synthesis of uncontrolled amounts of TNF- $\alpha$, superoxidanions and $\mathrm{NO}$, resulting in the formation of a "vicious circle", which further enhances neurotoxicity and leads to chronic neuroinflammation (Italiani \& Boraschi, 2014; Lan et al., 2018).

Antigen-presenting cells (APC) are known to play a key role in the activation of autoreactive myelin-specific $\mathrm{T}$ lymphocytes in the periphery (Lester \& Li, 2014; Xie et al., 2015). To activate T cells, two main types of signals from APC are required: 1) presentation of the antigen connected to MHC on the APC surface to T cell receptor (TCR) and 2) interaction between cosignal molecules on the $\mathrm{T}$ cell surface and their corresponding 
ligands on APC. According to their functions, cosignal molecules can be divided into costimulatory molecules that stimulate TCR reactions (CD28 - CD80/CD86 [B7-1/B7-2], CD154 [CD40L] - CD40, CD278 $\mathrm{CD} 275$ ), and coinhibitory molecules that inhibit $\mathrm{T}$ cell activation (CD152 [CTLA-4] - CD80/CD86, PD-1 - PD-L1/PD-L2) (Wiesemann et al., 2008). In experimental autoimmune encephalomyelitis (EAE), an animal model for MS, changes in positive and negative costimulatory signals have been shown to play a key role in disease onset and activity (Francisco et al., 2010).

Recent studies have shown that binding to PD-1 (programmed cell death 1) its ligands PD-L1 or PD-L2 mediate the inhibition of T cell responses. In patients with MS, PD-1 gene polymorphism was associated with progressive course of disease, and IFN- $\beta$ therapy caused activation of PD-L1 expression on APC. Schreiner (2004) showed that PD-L1 blockade on human monocytes and dendritic cells led to increased $T$ cell proliferation and secretion of Th1 cytokines in vitro. In the EAE model, it was shown that PD-1, PD-L1 and PD-L2 were expressed on brain infiltrating cells, and PD-1 blockade increased the severity of EAE (Francisco et al., 2010). Changes in the functional state of mononuclear phagocytes in MS and understanding their role in disease pathogenesis could be investigated by study of the effects of exogenous and endogenous agents on these cells in vitro. It can be performed by stimulating mononuclear phagocytes with E. coli lipopolysaccharide, which is a toll-like receptor 4 (TLR4) agonist, and a single-stranded RNA preparation as a TLR7/8 agonist. Stimulation of monocytes through toll-like receptors (TLR) leads to changes in functional status and metabolic orientation; this is manifested through cytokine synthesis, expression of costimulatory molecules and changes in arginine metabolism, which are necessary for homeostasis maintaining (Herzmann et al., 2017).

The pathogenic role of L-arginine metabolites in MS is the subject of active research, concentration of some of them in cerebrospinal fluid and plasma are used as biomarkers of disease activity (Adiele \& Adiele, 2019). Elevated levels of NO in the cerebrospinal fluid correlate with axonal damage, clinical disorders, radiological markers, as well as with the rate of disability (Okada et al., 2017). Exacerbation of MS is accompanied by decrease in arginase activity and increase in the concentration of nitric oxide in plasma and cerebrospinal fluid in patients with isolated syndrome and relapsing-remitting MS (Ljubisavljevic et al., 2012). The direction of L-arginine metabolism is an important characteristic of mononuclear phagocytes, which reflects their functional and phenotypic features (Rath et al., 2014; Sica et al., 2015). The NO-forming pathway of arginine is connected mainly with inducible NO-synthase (iNOS), which forms nitric oxide (NO) and L-citrulline. The arginase pathway is realized with type I and II isoforms of arginase which produce L-ornithine and urea. NF-kBmediated mechanisms play a central role in the regulation of L-arginine metabolism. Stimulation of TLR, in particular TLR4, can cause activation of the NF- $\mathrm{KB}$ pathway, which leads to an increase in the first expression of the iNOS gene, and secondly - the production of proinflammatory cytokines (IL- $1 \beta, T N F-\alpha$, etc.), which are also inducers of iNOS. An example of the mechanism of negative regulation of NF- $\mathrm{kB}$ pathway activity is the accumulation of nitric oxide, which leads to a decrease in iNOS gene expression. The study of NO-synthase and arginase pathways of L-arginine metabolism of mononuclear phagocytes in MS and EAE (Weissert, 2016; Lubina-Dąbrowska et al., 2017) showed that stimulation of mononuclear phagocytes by inducers of proinflammatory (lipopolysaccharide and IFN- $\gamma$ ) or anti-inflammatory (IL-4) response, leads to pronounced changes in gene expression and activity of iNOS or arginases, and, accordingly, the production of significant amounts of oxy nitrogen or L-ornithine (Rath et al., 2014). Due to this, the determination of NO production and arginase activity has become the "gold standard" in the study of functional polarization of tissue macrophages and peripheral blood monocytes of mice (Reiner, 2009). However, significant differences in the biology and reactions of mononuclear phagocytes of mice of different lines, other animals, and humans leave open the question of the possibility of using these methods to determine the functional and phenotypic features of peripheral blood monocytes in patients, and in general the suitability of animals for modeling activation of mononuclear human phagocytes (Murray et al., 2014; Thomas \& Mattila, 2014). In addition, existing data on the activity of iNOS and arginases of mononuclear phagocytes in different types of MS indicate a wide range of reactions by these cells in response to the action of certain activators, and require further study (Christophi et al., 2009; Bogdan, 2015).

The aim of the investigation was to study the functional and metabolic features of intact and stimulated mononuclear phagocytes in patients with different clinical forms of MS.

\section{Materials and methods}

Immunological studies were conducted in the Laboratory of Clinical Immunology and Allergology of the State Institution "Mechnikov Institute of Microbiology and Immunology of the National Academy of Medical Sciences of Ukraine".

We examined 98 patients with a verified diagnosis of "multiple sclerosis" established in the Department of Neuroinfections and Multiple Sclerosis of the State Institution "Institute of Neurology, Psychiatry and Addiction of the National Academy of Medical Sciences of Ukraine". All participants gave their voluntary written consent to take part in the study.

All patients were divided into two groups depending on clinical course of the disease: 1) a group of patients with relapsing-remitting MS (RRMS), $\mathrm{n}=66$ (13 men and 53 women), the mean age of patients was $33.2 \pm 9.3$ years; included patients with a disease duration of at least 12 months who had at least one exacerbation or one paramagnetic contrast center on MRI 12 months prior to randomization; 2) a group of patients with progressive MS (PMS), $\mathrm{n}=32$ (9 men and 23 women), the mean age was $41.3 \pm 10.2$ years. In PMS, remission after the onset is absent, due to formation of the stage of progression and occurred either through the stabilization stage after the onset, or immediately after the onset. For six months before the collection of biological material, patients had not received any therapy with the drugs that could modify the course of the disease.

The control group included 27 healthy individuals $(9$ men and 18 women) with a mean age of $30.1 \pm 8.2$ years. Criteria for inclusion in the control group were the absence of acute infections for at least 1 month before taking biological material, the absence of chronic inflammatory, allergic and autoimmune diseases.

Isolation of peripheral blood mononuclear cells was performed by the method of Repnik et al. (2003), adapted to small blood volumes. Separation was performed using a double gradient of Percoll (Sigma, USA). To prepare $100 \mathrm{~mL}$ of Percoll iso-osmotic solution, $41.5 \mathrm{~mL}$ of Percoll with $\rho=1.131 \mathrm{~g} / \mathrm{cm}^{3}$ was dissolved in $48.5 \mathrm{~mL}$ of distilled water, $10 \mathrm{~mL}$ of $1.5 \mathrm{M} \mathrm{NaCl}$ solution was added and mixed thoroughly. To prepare $100 \mathrm{~mL}$ of Percoll hyperosmotic solution, $48.5 \mathrm{~mL}$ of Percoll with $\rho=$ $1.131 \mathrm{~g} / \mathrm{cm}^{3}$ was dissolved in $41.5 \mathrm{~mL}$ of distilled water, $10 \mathrm{~mL}$ of $1.6 \mathrm{M}$ $\mathrm{NaCl}$ solution was added and also mixed thoroughly.

To isolate cells of the monocyte fraction, $1 \mathrm{~mL}$ of the prepared mononuclear suspension was carefully layered on $3 \mathrm{~mL}$ of Percoll hyperosmotic solution and centrifuged at $580 \mathrm{~g}$ for 15 minutes at $+20^{\circ} \mathrm{C}$. The cell layer was carefully removed from the phase interface using a plastic Pasteur pipette, resuspended in RPMI- 1640 medium to $12 \mathrm{~mL}$ and centrifuged at $350 \mathrm{~g}$ for 7 minutes at $+20^{\circ} \mathrm{C}$. After removal of the supernatant, the precipitate was resuspended in $1 \mathrm{~mL}$ of RPMI-1640 medium, transferred to a new tube, RPMI-1640 medium was added, bringing the volume to $12 \mathrm{~mL}$ and centrifuged at $350 \mathrm{~g}$ for 7 minutes at $+20^{\circ} \mathrm{C}$. After centrifugation the supernatant was removed, and the pellet was resuspended in $3 \mathrm{~mL}$ of complete RPMI medium (10\% fetal calf serum, $90 \%$ RPMI-1640 medium supplemented with $0.2 \mathrm{mM}$ L-glutamine, $0,5 \mathrm{mM}$ HEPES $\mathrm{pH}$ and $0.1 \%$ gentamicin). The resulting suspension was adjusted to $5 \cdot 10^{6}$ cells $/ \mathrm{mL}$ and divided by $0.1 \mathrm{~mL}$. The number of monocytes in the suspension, determined with anti-CD14 PE-labeled monoclonal antibodies (EXBIO Praha, Czech Republic), was $75 \%$ or more. Cell viability after trypan blue staining was at least $98 \%$. The relative content of CD80, CD86 and PD-L1 positive peripheral blood monocytes was determined with FITC-labeled monoclonal antibodies against CD80, PE-Cy7-labeled monoclonal antibodies against CD86 and APC labeled monoclonal antibodies against PD-L1 produced by EXBIO Praha (Czech Republic).

Cells of the monocyte fraction were cultured in four parallel series (the scheme of the study is shown in Figure 1): a) with the addition of E. coli lipopolysaccharide ("Sigma-Aldrich", USA) at concentration $1 \mu \mathrm{g} / \mathrm{mL}$ as a stimulator of TLR4, b) with the addition of a complex of 
resistant to nucleases guanine and uracil-enriched phosphothioate-modified riboxioligonucleotide and cationic lipid ssRNA40/LyoVec (Invivogen, USA) at concentration $1 \mu \mathrm{g} / \mathrm{mL}$ as a stimulator of TLR7/8, c) with the addition of IL -4 as an anti-inflammatory factor ("Sigma-Aldrich", USA) at concentration of 10 units $/ \mathrm{mL}$, d) control samples, cultured without an inducer. The monocytes' suspension was cultured in 96-well plates at concentration $10^{5}$ cells $/ \mathrm{mL}$ in a final volume of $0.2 \mathrm{~mL}$ for 24 hours at $37^{\circ} \mathrm{C}$ in an atmosphere of $5 \% \mathrm{CO}_{2}$.

After incubation, the suspension was centrifuged at $350 \mathrm{~g}$ for $7 \mathrm{mi}-$ nutes at $+20^{\circ} \mathrm{C}$, the precipitate was used to determine the expression of cosignal molecules and arginase activity (series a, b, d), and the supernatant-to determine the concentration of nitrites (series a, b, c, d).

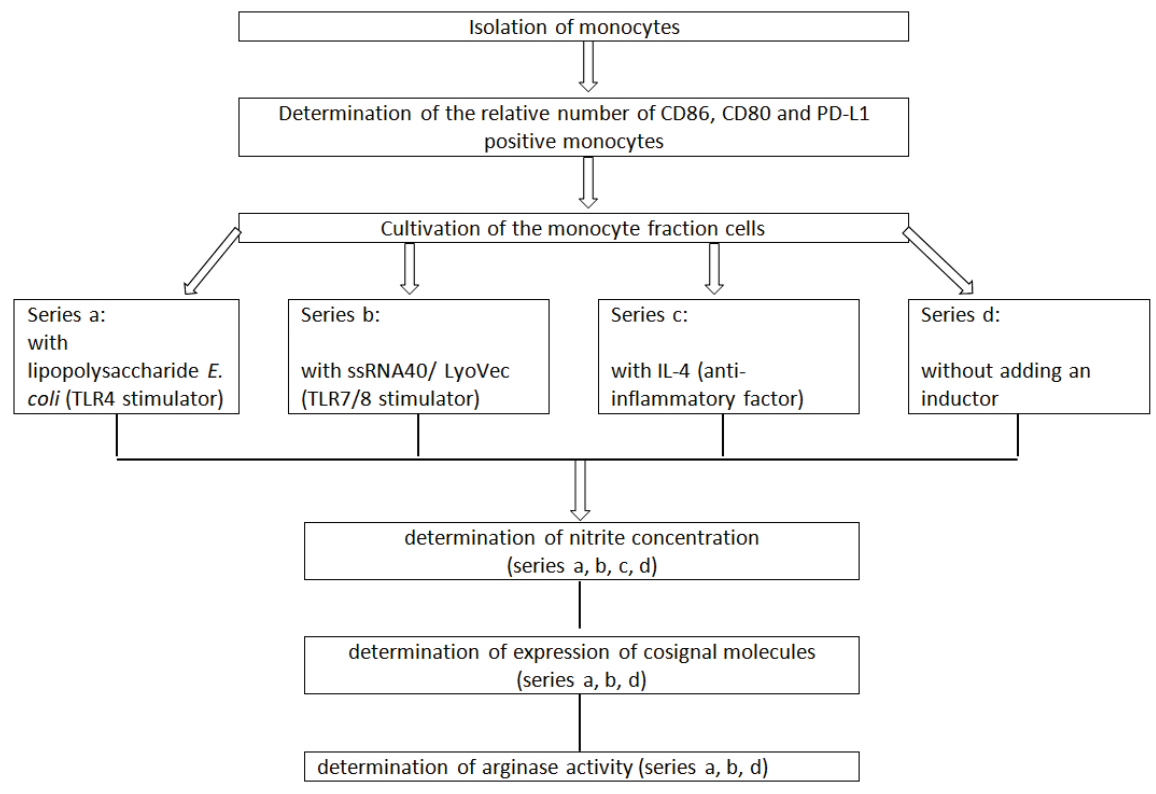

Fig. 1. The scheme of the research

Arginase activity in cell lysates was determined by the method of Classen et al. After removing the supernatant from the wells of the plate, the pellets with cultured cells were carefully washed with $0.2 \mu \mathrm{L}$ of phosphate buffered saline $\left(\mathrm{NaCl}-8 \mathrm{~g} / \mathrm{L} ; \mathrm{KCl}-0.2 \mathrm{~g} / \mathrm{L} ; \mathrm{Na}_{2} \mathrm{HPO}_{4} \times 2 \mathrm{H}_{2} \mathrm{O}-\right.$ $0.76 \mathrm{~g} / \mathrm{L} ; \mathrm{KH}_{2} \mathrm{PO}_{4}-0.2 \mathrm{~g} / \mathrm{L}$ ), after careful removal of the washing solution $0.1 \mathrm{~mL}$ of a $0.1 \%$ aqueous solution of Triton X-100 (Sigma-Aldrich, USA) was added to the wells and left for 15 minutes on a shaker for cell lysis. Then $0.1 \mathrm{~mL}$ of $50 \mathrm{mM}$ Tris- $\mathrm{HCl}$ buffer $(\mathrm{pH}=7.5$; Sigma-Aldrich, USA) and $0.01 \mathrm{~mL}$ of $100 \mathrm{mM} \mathrm{MnCl} 2$ solution were added. $0.1 \mathrm{~mL}$ of lysate from the wells was transferred to "safelock" tubes and incubated for 7 minutes at $56^{\circ} \mathrm{C}$ for activation of arginase. Then, $0.1 \mathrm{~mL}$ of a $0.5 \mathrm{M}$ arginine solution ( $\mathrm{pH}=9.7$; Sigma-Aldrich, USA) was added to each tube and incubated at $\mathrm{t}=+37^{\circ} \mathrm{C}$ for 120 minutes (series without adding an inductor, with the addition of LPS and ssRNA40/LyoVec) and $60 \mathrm{mi}-$ nutes (series with the addition of $\mathrm{IL}-4$ ). The reaction was stopped by adding $0.8 \mathrm{~mL}$ of stop reagent $\left(\mathrm{H}_{3} \mathrm{PO}_{4}, \mathrm{H}_{2} \mathrm{SO}_{4}\right.$ and distilled water in a ratio of $1: 3: 7$ ) to test tubes, and $0.9 \mathrm{~mL}$ to test tubes with $0.1 \mathrm{~mL}$ of standard solutions containing $0,7.5,15.0,30.0,45.0$ and $60.0 \mu \mathrm{g}$ of urea. To each tube was added $40 \mu \mathrm{L}$ of $6 \%$ alcoholic solution of $\alpha$-isonitrosopropiophenone and vortexed for 5 seconds, then sequentially incubated for 30 minutes at $\mathrm{t}=+95^{\circ} \mathrm{C}$ and for 30 minutes at $\mathrm{t}=+4^{\circ} \mathrm{C}$. After incubation, the urea content in the samples was determined on Stat-Fax 303 plus (USA) with a filter installed at $545 \mathrm{~nm}$.

The concentration of nitrites in the supernatants was determined by the method of Gris (Reiner, 2009). $0.1 \mathrm{~mL}$ of culture supernatants was transferred to the wells of a 96-well plate, mixed with $0.1 \mathrm{~mL}$ of Gris reagent ( $1 \%$ sulfonamide, $0.1 \%$ 1-naphthylenediamine in $2.5 \%$ phosphoric acid solution) and incubated for $30 \mathrm{~min}$ at $\mathrm{t}=+20^{\circ} \mathrm{C}$ in darkness. Absorption was read on Stat-Fax 303 plus (USA) with a $545 \mathrm{~nm}$ filter installed and the nitrite concentrations were calculated according to a standard calibration curve. The results obtained were presented in $\mu \mathrm{mol} \mathrm{NO} / 10^{5}$ cells.

Statistical processing of the obtained data was performed using Statistica 11.0 (StatSoft, Inc.) and Xlstat 19.6 (Addinsoft). The normality of the data distribution in the groups was checked using the W-Shapiro-Wilk test. The significance of the differences in the studied samples was determined with z-test with Holm-Bonferroni correction to exclude the familywise error rate (Microsoft R Open, https://mran.microsoft.com/rro). The critical value to assess the significance across these findings was 0.05 .

\section{Results}

Patients with PMS had a more severe course of the disease with rapid progression of clinical symptoms and the formation of persistent neurological deficit, increased score on the expanded disability status scale (EDSS), which was $5.5 \pm 1.0$ points, and no remission. Patients with RRMS showed a more benign clinical course with different degrees of severity and rate of formation of clinical symptoms, which alternated with remissions of varying duration. According to the EDSS scale, the disability rate of these patients was $3.0 \pm 1.4$ points.

It is known that the state of activity of peripheral blood monocytes can directly affect the course of the disease. The relative number of monocytes expressing CD86 and CD80 (Fig. 2) in patients with RRMS did not have significant differences relative to the control group. In patients with PMS, the relative number of CD86+ cells on intact peripheral blood monocytes (Fig. 2) was significantly higher than in healthy individuals $(87.9 \pm 5.4 \%$ vs. $74.4 \pm 6.4 \%, \mathrm{P}<0.05)$ while, in contrast, expression of PD-L1 molecules was significantly reduced $(35.9 \pm 3.8 \%$ vs. $52.1 \pm 6.1 \%$, $\mathrm{P}<0.05)$. In the group of patients with RRMS, the figures were quite different, the rate of expression of PD-L1 molecules of intact peripheral blood monocytes was increased $(62.2 \pm 5.6 \%$ vs. $52.1 \pm 6.1 \%$ in the control, $\mathrm{P}<0.05$ ), while expression of CD86+ showed a slight tendency to increase and did not differ significantly from figures of PMS and control groups. It should also be noted that the expression of PD-L1 was significantly different when compared between groups of patients $(\mathrm{P}<0.05)$. The relative number of $\mathrm{CD} 80+$ cells was quite similar in all groups; it was $5.4 \pm 1.8 \%$ in the PMS group, somewhat less in RRMS patients and $5.6 \pm$ $1.3 \%$ in the control group $(\mathrm{P}>0.05)$.

In the next part of the study, the dependence of the expression level of cosignal molecules on the characteristics of stimulus was investigated, which revealed differences in the reserve capacity of the monocytes from peripheral blood in patients with different clinical courses. The classic targets for TLR4 stimulation of mononuclear phagocytes are LPS. Therefore, incubation with LPS was used to study and analyze the expression of cosignant molecules of these cells.

Stimulation of TLR4 and TLR7/8 by culturing monocytes for 24 hours in the presence of lipopolysaccharide E. coli and ssRNA40/Lyo Vec at a concentration of $1 \mu \mathrm{g} / \mathrm{mL}$ produced a pronounced and stable activation of the cells (Fig. 3, 4). 




Fig. 2. The relative number of CD86, CD80 and PD-L1-positive peripheral blood monocytes in patients with relapsing-remitting multiple sclerosis (RRMS, $n=66$ ), progressive multiple sclerosis $(P M S, n=32)$ and healthy individuals (control, $\mathrm{n}=27, \%, \mathrm{x} \pm \mathrm{SD}$ ): * $-\mathrm{P}<0.05$ when compared with the control group; ${ }^{\#}-\mathrm{P}<0.05$ when compared between groups of patients

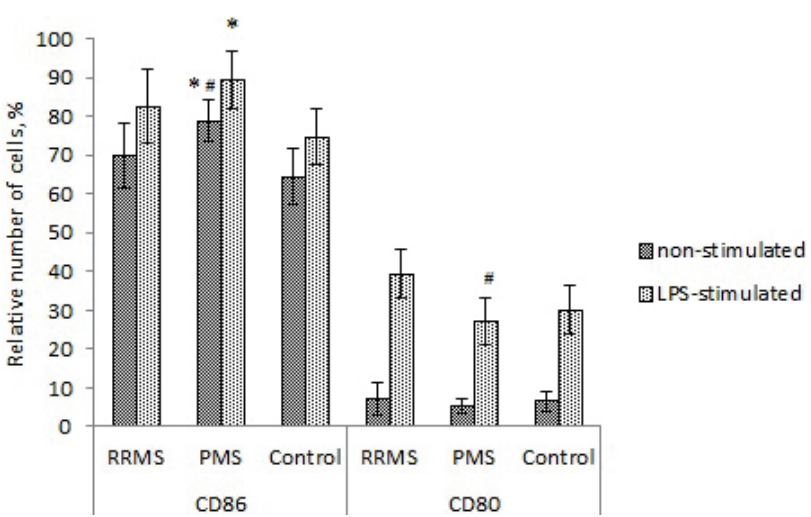

Fig. 3. The relative number of CD 86 and CD 80 positive peripheral blood monocytes in patients with relapsing-remitting multiple sclerosis (RRMS,

$\mathrm{n}=66$ ) and progressive multiple sclerosis (PMS, $\mathrm{n}=32$ ) without

stimulant and upon LPS stimulation (TLR4 agonists) compared with healthy individuals (control, $\mathrm{n}=27, \%, \mathrm{x} \pm \mathrm{SD}$ ):

* $-\mathrm{P}<0.05$ when compared with the control group;

\# $-\mathrm{P}<0.05$ when compared between groups of patients

The level of CD86 expression on monocytes in the RRMS group when stimulated with TLR4 agonists increased from $69.9 \pm 8.3 \%$ to $82.6 \pm 9.5 \%$ and the expression level of CD 80 increased by $32.4 \%$ from baseline - and amounted to $39.5 \pm 6.3 \%$, but it was at the level of control group. Incubation of monocytes with LPS led to an increase in the expression of CD86 on monocytes in the group of PMS $(89.5 \pm 7.6 \%$ vs. $74.8 \pm$ $7.3 \%$ in the control, $\mathrm{P}<0.05$ ). The expression level of CD80 molecules in the PMS group increased 5 times from baseline and amounted to $27.1 \%$. It is important to note that the relative number of CD80 positive monocytes was significantly lower than in the RRMS group (Fig. 3).

Stimulation with TLR7/8 agonists of peripheral blood monocytes from patients of RRMS and PMS groups did not lead to significant changes in CD86 and CD80 expression relative to the control group and when compared between groups (Fig. 4).

When stimulated with TLR4 (LPS) agonists, an increase in the expression level of the PD-L1 molecule was observed in the RRMS group $(54.5 \pm 9.0 \%$ vs. $41.2 \pm 5.3 \%$ in the control, $\mathrm{P}<0.05)$. The level of PD-L1 expression in the PMS group after incubation was significantly lower compared with the control group $(25.8 \pm 3.8 \%$ vs. $31.3 \pm 6.0 \%)$ and compared with the RRMS group $(\mathrm{P}<0.05$, Fig. 5a)

Incubation of monocytes in the presence of ssRNA40/LyoVec, which is a TLR7/8 agonist, resulted in the increase in PD-L1 expression in the patients with RRMS reliable relative to control $(61.0 \pm 8.0 \%$ vs. $42.2 \pm$ 9.0\%) and decreased expression in patients with PMS relative to control $(25.8 \pm 3.8 \%$ vs. $32.1 \pm 4.4 \%)$ and the RRMS group ( $\mathrm{P}<0.05$, Fig. $5 b)$. In the second part of the work we compared the metabolic orientation of arginine pathways in the monocytes from peripheral blood of patients with
RRMS and PMS. In the groups of patients, moderate basal activity of arginases was detected, very close to control level $(\mathrm{P}>0.05$ relative to control). The unstimulated NO production in all groups was $<0.1 \mu \mathrm{mol}$ $\mathrm{NO} / 10^{5}$ cells, which indicates the predominance of the arginase pathway of L-arginine metabolism in intact cells of the monocyte fraction of peripheral blood mononuclear cells of all investigated groups.



Fig. 4. The relative number of $C D 86$ and $C D 80$ positive peripheral blood monocytes in patients with relapsing-remitting multiple sclerosis $($ RRMS, $\mathrm{n}=66$ ) and progressive multiple sclerosis $(\mathrm{PMS}, \mathrm{n}=32)$ without stimulant and upon ssRNA40/LyoVec stimulation (TLR7/8 agonists) compared with healthy individuals (control, $\mathrm{n}=27, \%, \mathrm{x} \pm \mathrm{SD}$ )



Fig. 5. Relative number of PD-L1 positive peripheral blood monocytes in patients with relapsing-remitting multiple sclerosis (RRMS, $n=66$ ) and progressive multiple sclerosis (PMS, $n=32$ ) without stimulant and upon LPS stimulation (TLR4 agonists) (a) and ssRNA40/LyoVec stimulation (TLR7/8 agonists) (b) compared with healthy individuals (control, $\mathrm{n}=27, \%, \mathrm{x} \pm \mathrm{SD}): *-\mathrm{P}<0.05$ when compared with the control group; ${ }^{\#}-\mathrm{P}<0.05$ when compared between groups of patients

Table 1

Arginase activity of peripheral blood monocytes of patients with relapsing-remitting multiple sclerosis (RRMS, $n=66$ ), progressive multiple sclerosis (PMS, $\mathrm{n}=32$ ) and healthy individuals (control, $\mathrm{n}=27, \mathrm{mU} / 10^{5}$ cells, $\mathrm{x} \pm \mathrm{SD}$ )

\begin{tabular}{lccc}
\hline \multirow{2}{*}{ Series } & \multicolumn{2}{c}{ Patients group } & \multirow{2}{*}{ Control } \\
\cline { 2 - 3 } & RRMS & PMS & \\
\hline $\mathrm{n}$ & 66 & 32 & 27 \\
Non-stimulated cells & $2.64 \pm 0.28$ & $2.50 \pm 0.49$ & $2.37 \pm 0.51$ \\
LPS-stimulated cells & $10.76 \pm 2.11^{*}$ & $6.51 \pm 0.95^{*}$ & $12.48 \pm 1.54$ \\
ssRNA40/LyoVec-stimulated cells & $7.25 \pm 1.06$ & $8.74 \pm 2.23$ & $5.72 \pm 0.80$ \\
IL-4-stimulated cells & $3.57 \pm 0.79$ & $3.20 \pm 1.01$ & $4.05 \pm 1.42$ \\
\hline
\end{tabular}

Notes: ${ }^{*}-\mathrm{P}<0.05$ when compared with the control group (according to the results of z-test taking into account Holm-Bonferroni correction).

Cultivation for 24 hours in the presence of a TLR4 agonist led to an increase in arginase activity - 4.1 times in the RRMS group, 2.6 times in the PMS group and 5.3 times in the control group $(\mathrm{P}<0.05)$ compared with non-stimulated cells. Stimulation of TLR7/8 also caused an increase in arginase activity -2.7 times in the RRMS group, 3.5 times in the PMS group and 2.4 times in the control $(\mathrm{P}<0.05)$. Compared with the control group, the increase in arginase activity in monocytes of patients with MS was less pronounced with stimulation of LPS and IL 4 , but more pronounced with stimulation of ssRNA. Stimulation of cells with LPS and ssRNA40/LyoVec did not lead to a significant increase in nitric oxide pro- 
duction, the content of which in all samples remained below $1 \mu \mathrm{mol}$ $\mathrm{NO} / 10^{5}$ cells.

\section{Discussion}

The generally accepted concept assumes polarization of macrophages into two opposite activation states: pro-inflammatory, or classically activated (M1), and anti-inflammatory, or alternatively activated (M2) macrophages. Later, M2 phagocytes were divided into M2a, M2b, and M2c phagocytes to differentiate phenotypes caused by different stimuli. At the same time, macrophages and microglia retain their plasticity after polarization, indicating that they can adapt to changing environments (Bogie et al., 2014). In neuroinflammatory and neurodegenerative disorders, these cells are exposed to numerous soluble factors in the periphery and CNS, some of these mediators play an important role in changes in their physiology. It becomes clear that macrophages and microglia, depending on the microenvironment, could display a spectrum of activated phenotypes, rather than discrete stable ones.

The data obtained in our work indicate that in MS patients monocytes of peripheral blood manifest signs of pro-inflammatory as well as anti-inflammatory activity depending on the clinical course of the disease. The expression of costimulatory molecules CD80 and CD86, which are involved in the realization of intercellular synapses, is an important characteristic of the state of mononuclear phagocytes, namely their participation in lymphocytes' activation and antigens' presentation.

In patients with progressive disease, the relative number of CD86+ cells was significantly higher than in patients with RRMS and healthy individuals, this corresponds to the results of Wiesemann et al. (2008), who found that CD86 expression levels in monocytic cells were significantly higher than ones in RRMS patients. There was also an increased expression of CD80+ in the active areas of MS lesions, as well as on monocytes and $\mathrm{B}$ cells of cerebrospinal fluid and peripheral blood. In our study we did not find significant differences in expression of CD80+ in monocytes in MS patients relative to indicators in the control group, as well as when comparing groups of RRMS and PMS. It may be connected with more severe course of the disease in Wiesemann's investigation.

An important function in the regulation of immune homeostasis and in the maintenance of peripheral tolerance through secondary costimulatory signaling of activated lymphocytes is played by PD-1 and its ligands (PD-L1 and PD-L2) - transmembrane proteins that have different patterns of expression and differ in their affinity. PD-1 and its ligands protect against potentially pathogenic autoreactive effector $\mathrm{T}$ cells by simultaneously affecting two mechanisms of peripheral tolerance: stimulating the development and function of Treg, and by directly inhibiting potentially pathogenic autoreactive T cells in the periphery. PD-1-induced Treg cells can also help maintain immune homeostasis by maintaining a T-cell activation threshold high enough to protect against autoimmune processes. Expression of PD-L1 on non-hematopoietic as well as hematopoietic cells promotes the development of Tregs and enhances the function of Tregs in lymphoid organs and tissues that are the target of autoimmune attack (Francisco et al., 2010).

In our study, pro-inflammatory markers could include increased expression of CD86 and CD80, while anti-inflammatory - increased expression of PD-L1 and increased arginase activity. Low arginase activity is associated with polarization of monocytes in M1 (Nahrendorf \& Swirski, 2016; Rodriguez et al., 2017), given this decreased arginase activity in monocytes of PMS patients (Table 1) under LPS treatment may be considered as an indirect proinflammatory sign.

It is important to note that in our study, the percentage of PD-L1positive monocytes in the PMS group was lower than in the control group and compared with the RRMS group, which in combination with increased level of CD86 expression can be considered as signs of monocytes polarization trend in M1 direction in PMS patients. In RRMS patients, on the contrary, we found increased expression of PD-L1 marker, it can be regarded as a sign of enhanced anti-inflammmatory activity. Unfortunately, it was not confirmed by other markers as was the case in PMS patients. In patients with PMS, three markers were found that may indicate polarization of peripheral blood monocytes in the M1 direction - increased expression of CD86, decreased expression of CD80, and a decrease in argi- nase activity under LPS loading, while an increase in NO formation was not detected, and the arginase activity after ssRNA40/LyoVec treatment tended to increase. On the whole, this allows us to speak about the polarization trend towards M1. In RRMS patients polarization signs are less clear.

The presence of different forms of macrophage activation in patients with different severity of the disease corresponds to data on the state of cells in the brain lesion in MS patients. In early lesion microglia and macrophages show dominant expression of proinflammatory markers involved in phagocytosis (CD68), antigen presentation (MHC class I and II, CD86), and reactive oxygen species (ROS) production (eg, p22phox). In the later stages of active lesions, cells switch to an intermediate phenotype and co-express proinflammatory markers with other antigens associated with M2 activation (CD206 and CD163). Patterns of microglia and macrophage activation are qualitatively similar in new demyelinating lesions of early MS and with the slow spread of lesions of progressive MS (O’Loughlin et al., 2018).

Deciphering the factors that regulate the changes of macrophages and microglia in neurological disorders is very important for understanding pathogenic and neuroprotective pathways and developing new therapeutic agents. New results on these factors, such as soluble mediators and intercellular interactions, have provided a crucial insight into the mechanisms governing the spatiotemporal dependence of phagocyte physiology in CNS pathologies. Our next task was to present various factors influencing the functional properties of a subset of macrophages during neuroinflammation and neurodegeneration.

Stimulation with TLR4 and TLR7/8 agonists revealed differences in the reserve capacity of cells of the monocyte fraction of peripheral blood mononuclear cells in patients with different clinical course. In particular, TLR4-mediated activation of monocytes obtained from patients with PMS was accompanied by significantly increased expression of CD86 (relative to control).

Increased reserve capacity for LPS- and ssRNA-induced PD-L1 expression in patients with RRMS and decreased reserve capacity in patients with PMS, indicating depletion of producer cells due to prolonged antigenic stimulation. Thus, changes in the expression of cosignal molecules in the brain and body fluids indicate the important role of these mediators in influencing the identity of macrophages and microglia in inflammatory conditions of the CNS.

Previously (Tupotilov \& Kolyada, 2018) we showed differences in the subpopulation composition of monocytes with respect to basal and LPS and ssRNA40/LyoVec-induced cytokine production in vitro by cells of the monocyte fraction of peripheral blood mononuclear cells in patients with different clinical course of MS. Proinflammatory and regulatory cytokines, including IFN $\gamma$, IL-1 $1 \beta$, TNF $\alpha$, IL-10 and IL-12, are abundant in the bloodstream and CNS of MS patients. These cytokines are well known for their ability to stimulate the inflammatory transcription profile in phagocytes. The addition of TLR4 and TLR7/8 agonists in the cultivation of monocytes from healthy people led to a marked increase in the production of TNF- $\alpha$ and IL-1 $\beta$. Stimulation of IL- $1 \beta$ production with the addition of ssRNA40/LyoVec in patients with advanced MS was stronger compared with LPS. At the same time, in patients with MS, the reserve capacity of monocytes to stimulated production of TNF- $\alpha$ and IL- $1 \beta$ in response to the addition of LPS and single-stranded RNA was reduced relative to control, especially in patients with recurrent MS $(\mathrm{P}<0.05)$. This may indicate changes in the subpopulation of cells producing these cytokines on the one hand, and on the other - the "depletion" of these cells, the presence of disorders of MyD88-mediated signaling and activation of NF- $\mathrm{kB}$.

In our second study (Vdovichenko et al., 2020), the serum cytokine profile of patients with MS in different periods of disease activity allowed us to establish a relationship between the state of the immune system, MS progression and the degree of disability of patients on the EDSS scale. In patients with RRMS in the acute stage, there were elevated levels of IFN- $\gamma$, IL-1 $\beta$ and IL-12, while in the group of patients with RRMS in the stage of clinical and radiological remission, increased production of proinflammatory cytokines was accompanied by a compensatory increase in IL-10. In patients with progressive type of MS, regardless of the stage of progression, there was a more pronounced cytokine imbalance on the background of reduced IL-10. This cytokine profile indicates a decrease in the reserve capacity of the regulatory link of humoral immunity, while cli- 
nical data confirm this by high values of disability on the EDSS scale at the stage of steady progression of the disease.

In the blood of healthy people, the physiological relationship between subpopulations of monocytes is maintained, which may change in pathological conditions (Murray, 2017). The role of individual subpopulations in the formation and replenishment of the pool of mononuclear phagocytes in the CNS is still insufficiently defined (Okabe \& Medzhitov, 2016). Many pathological conditions of different organ localization are associated with one or another metabolic shift of macrophage mononuclear phagocytes, which does not meet the physiological needs of a particular tissue (Sica et al., 2015). Arginine metabolism plays an important role in the regulation of macrophage activation. Mononuclear phagocytes are characterized by the ability to acquire a wide range of functional states depending on the stimuli that cause their activation. Each of these polarization states involves the activation of metabolic responses necessary to maintain homeostasis. Arginine metabolism is a central regulator of macrophage activation. Two opposite pathways cause the metabolic conversion of arginine in these cells. Nitric oxide synthase converts arginine to nitric oxide, the radicals of which cause the cytotoxic effect of proinflammatory (M1) macrophages, and arginase to proline and polyamines, which play an important role in collagen synthesis and cell proliferation, thus causing anti-inflammatory function of macrophages (Rodriguez et al., 2017).

In groups of patients with relapsing-remitting and progressive type of multiple sclerosis, we found moderate basal activity of arginases of intact monocytes, the level of which did not depend on the subpopulation composition of the cell fraction. Stimulation of monocytes by TLR4 and TLR7/8 agonists was accompanied by an increase in arginase activity, which was more pronounced in patients with a recurrent type of multiple sclerosis when culturing cells of the monocyte fraction with LPS. In patients with progressive disease, the arginase activity of monocytes when cultured with ssRNA40/LyoVec was significantly reduced relative to control and its level was also negatively correlated with the number of CD16+ cells in the fraction $(\mathrm{r}=-0.416 ; \mathrm{P}<0.05)$. Basal and stimulated nitric oxide production in all groups was $<0.1 \mu \mathrm{mol} \mathrm{NO} / 10^{5}$ cells, indicating a predominance of the arginase pathway of L-arginine metabolism in intact and activated after 24 hours of cultivation in the presence of LPS or ssRNA40/LyoVec monocyte cells in peripheral blood. Cultivation of cells of the monocyte fraction of peripheral blood mononuclear cells in patients with relapsing-remitting and progressive multiple sclerosis in the presence of IL-4 at a concentration of $50 \mathrm{U} / \mathrm{mL}$ also did not cause significant changes in both arginase activity and $\mathrm{NO}$ production. Data on the functioning of the arginase and NO-synthase pathways of L-arginine metabolism of intact and TLR4- and TLR7/8-activated peripheral blood monocytes in patients with multiple sclerosis indicate a subpopulation-dependent nature of differences in arginase activity in patients with different clinical course of the disease.

The results of our work indicate a tendency to activate peripheral blood monocytes in patients with multiple sclerosis with their polarization in the M1 direction with increased arginase activation with TLR7/8 stimulation and decreased - with TLR4 stimulation, especially in patients with progressive disease.

Our data are consistent with the results of other studies (Munder et al., 2005; Mattila et al., 2013; Rath et al., 2014; Okada et al., 2017), which also did not confirm the ability of monocytes/macrophages to any significant production of nitric oxide. Shin (2012) showed that NO production in patients with MS is significantly reduced compared to mice and rats. In the work of Rath et al. (2014) showed the presence of iNOS activity in monocytes infiltrating the active foci in the early stages of MS, while in chronic disease, infiltrated monocytes did not have iNOS activity. Basal and stimulated iNOS activity was detected only in CD16+ HLA-DR++ monocytes, the number of which was significantly higher in patients with MS.

At the same time, in other works activity of iNOS or arginases was observed (Babu, 2009; Mattila et al., 2013). Analyzing the causes of this phenomenon, various authors express the opinion that in addition to metabolic differences between human and rodent mononuclear phagocytes, it is also necessary to take into account the differences in the design of the work, the investigated pathology and features of the research methods used (Thomas \& Mattila, 2014). By analogy with mice, we can also assume the influence of genetic factors on the results obtained in different cohorts of humans (Ravasi et al., 2007). There is also evidence that, in contrast to mice and rats, in vitro stimulation of mononuclear phagocytes by LPS instead of activating arginine metabolism induces activation of the indoleamine-2,3-dioxygenase pathway of tryptophan metabolism (ThomaUszynski et al., 2001)

Christophi (2009) showed that, in contrast to mice, human IL-4 is able to stimulate rather than inhibit nitric oxide production. In our study, we evaluated the effect of IL- 4 on the direction of L-arginine metabolism of peripheral blood monocytes in patients with relapsing-remitting and progressive MS. Under conditions of 24-hour cultivation of monocytes with the addition of $\mathrm{IL}-4$ at a concentration of up to $50 \mathrm{U} / \mathrm{mL}$ we found no significant changes in both arginase activity and NO production.

Thus, data on arginase activity are contradictory and require further research involving improved approaches to determining the population composition of cells, which will clarify the features of the functional state of monocytes of different subpopulations and their pathogenetic role in the development and course of multiple sclerosis.

The model of TLR-dependent activation and assessment of the functional state of intact and activated mononuclear cells of the monocyte fraction in vitro of peripheral blood confirmed the existence of functionalphenotypic heterogeneity of cells associated with the type of clinical course of the disease. Differences in the mechanisms of activation of peripheral blood monocytes mediated by stimulation of TLR-like receptors of different types in patients with relapsing-remitting and progressive multiple sclerosis are revealed.

\section{Conclusions}

The functional state of mononuclear phagocytes, as representatives of innate immunity, affects the nature of the clinical course of multiple sclerosis. An in vitro study of TLR-mediated monocyte activation revealed an increase in the relative number of PD-L1-positive monocytes in patients with relapsing-remitting multiple sclerosis, while in patients with progressive disease, on the contrary, a decrease in the number of cells was revealed, while the relative number of CD86+ cells was significantly higher in comparison with sick RRMS and healthy persons.

The study of the arginase pathway of metabolism revealed a significant increase in arginase activity of monocytes upon stimulation of TLR4 and TLR7/8 in comparison with baseline, which was more pronounced in patients with RRMS during incubation with LPS, and in patients with PMS during incubation with ssRNA40/LyoVec.

The results show the signs of activation of the peripheral blood monocytes with the polarization trend towards M1 in patients with PMS. In RRMS patients the changes were less definite.

\section{References}

Adiele, R. C., \& Adiele, C. A. (2019). Metabolic defects in multiple sclerosis. Mitochondrion, 44, 7-14

Babu, S., Kumaraswami, V., \& Nutman, T. B. (2009). Alternatively activated and immunoregulatory monocytes in human filarial infections. The Journal of Infectious Diseases, 199(12), 1827-1837.

Baufeld, C., O’Loughlin, E., Calcagno, N., Madore, C., \& Butovsky, O. (2018). Differential contribution of microglia and monocytes in neurodegenerative diseases. Journal of Neural Transmission, 125(5), 809-826.

Bogdan, C. (2015). Nitric oxide synthase in innate and adaptive immunity: An update. Trends in Immunology, 36(3), 161-178.

Bogie, J. F., Stinissen, P., \& Hendriks, J. J. (2014). Macrophage subsets and microglia in multiple sclerosis. Acta Neuropathologica, 128(2), 191-213.

Christophi, G. P., Panos, M., Hudson, C. A., Christophi, R. L., Gruber, R. C., Mersich, A. T., Blystone, S. D., Jubelt, B., \& Massa, P. T. (2009). Macrophages of multiple sclerosis patients display deficient SHP-1 expression and enhanced inflammatory phenotype. Laboratory Investigation, 89(7), 742-759.

Francisco, L. M., Sage, P. T., \& Sharpe, A. H. (2010). The PD-1 pathway in tolerance and autoimmunity. Immunological Reviews, 236, 219-242.

Herzmann, N., Salamon, A., Fiedler, T., \& Peters, K. (2017). Lipopolysaccharide induces proliferation and osteogenic differentiation of adipose-derived mesenchymal stromal cells in vitro via TLR4 activation. Experimental Cell Research, 350(1), 115-122.

Italiani, P., \& Boraschi, D. (2014). From monocytes to M1/M2 macrophages: Phenotypical vs. functional differentiation. Frontiers in Immunology, 5, 514. 
Lan, M., Tang, X., Zhang, J., \& Yao, Z. (2018). Insights in pathogenesis of multiple sclerosis: Nitric oxide may induce mitochondrial dysfunction of oligodendrocytes. Reviews in the Neurosciences, 29(1), 39-53.

Lester, S. N., \& Li, K. (2014). Toll-like receptors in antiviral innate immunity. Journal of Molecular Biology, 426(6), 1246-1264.

Ljubisavljevic, S., Stojanovic, I., Pavlovic, R., Sokolovic, D., Pavlovic, D., Cvetkovic, T., \& Stevanovic, I. (2012). Modulation of nitric oxide synthase by arginase and methylated arginines during the acute phase of experimental multiple sclerosis. Journal of the Neurological Sciences, 318, 106-111.

Lubina-Dąbrowska, N., Stepien, A., Sulkowski, G., Dąbrowska-Bouta, B., Langfort, J., \& Chalimoniuk, M. (2017). Effects of IFN- $\beta 1 \mathrm{la}$ and IFN- $\beta 1 \mathrm{~b}$ treatment on the expression of cytokines, inducible NOS (NOS type II), and myelin proteins in animal model of multiple sclerosis. Archivum Immunologiae et Therapiae Experimentalis, 65(4), 325-338

Mammana, S., Fagone, P., Cavalli, E., Basile, M. S., Petralia, M. C., Nicoletti, F., Bramanti, P., \& Mazzon, E. (2018). The role of macrophages in neuroinflammatory and neurodegenerative pathways of Alzheimer's disease, amyotrophic lateral sclerosis, and multiple sclerosis: Pathogenetic cellular effectors and potential therapeutic targets. International Journal of Molecular Sciences, 19(3), 831.

Mattila, J. T., Ojo, O. O., Kepka-Lenhart, D., Marino, S., Kim, J. H., Eum, S. Y., Via, L. E., Barry, C. E. 3rd, Klein, E., Kirschner, D. E., Morris, S. M. Jr, Lin, P. L., \& Flynn, J. L. (2013). Microenvironments in tuberculous granulomas are delineated by distinct populations of macrophage subsets and expression of nitric oxide synthase and arginase isoforms. Journal of Immunology, 191(2), 773-784.

Munder, M., Mollinedo, F., Calafat, J., Canchado, J., Gil-Lamaignere, C., Fuentes, J. M., Luckner, C., Doschko, G., Soler, G., Eichmann, K., Müller, F. M., Ho, A. D., Goemer, M., \& Modolell, M. (2005). Arginase I is constitutively expressed in human granulocytes and participates in fungicidal activity. Blood, 105(6), 2549-2556.

Murray, P. J. (2017). Macrophage polarization. Annual Review of Physiology, 79, 541-566.

Murray, P. J., Allen, J. E., Biswas, S. K., Fisher, E. A., Gilroy, D. W., Goerdt, S., Gordon, S., Hamilton, J. A., Ivashkiv, L. B., Lawrence, T., Locati, M., Mantovani, A., Martinez, F. O., Mege, J. L., Mosser, D. M., Natoli, G., Saeij, J. P., Schultze, J. L., Shirey, K. A., Sica, A., \& Wynn, T. A. (2014). Macrophage activation and polarization: Nomenclature and experimental guidelines. Immunity, 41(1), 14-20.

Nahrendorf, M., \& Swirski, F. K. (2016). Abandoning M1/M2 for a network model of macrophage function. Circulation Research, 119(3), 414 417.

Okabe, Y., \& Medzhitov, R. (2016). Tissue biology perspective on macrophages. Nature Immunology, 17(1), 9-17.

Okada, K., Kobata, M., Sennari, Y., Iwanaka, Y., Hashimoto, T., Ohnari, K., \& Tahara, M. (2017). Levels of nitric oxide metabolites in cerebrospinal fluid correlate with cognitive impairment in early stage multiple sclerosis. Journal of Neurology, Neurosurgery, and Psychiatry, 88(10), 892-893.
O'Loughlin, E., Madore, C., Lassmann, H., \& Butovsky, O. (2018). Microglial phenotypes and functions in multiple sclerosis. Cold Spring Harbor Perspectives in Medicine, 8(2), a028993.

Rath, M., Müller, I., Kropf, P., Closs, E. I., \& Munder, M. (2014). Metabolism via arginase or nitric oxide synthase: Two competing arginine pathways in macrophages. Frontiers in Immunology, 5, 532.

Ravasi, T., Wells, C. A., \& Hume, D. A. (2007). Systems biology of transcription control in macrophages. BioEssays, 29(12), 1215-1226.

Reiner, N. E. (2009). Methods in molecular biology. Macrophages and dendritic cells. Methods and protocols. Preface. Methods in Molecular Biology, 531, v-vi.

Repnik, U., Knezevic, M., \& Jeras, M. (2003). Simple and cost-effective isolation of monocytes from buffy coats. Journal of Immunological Methods, 278, 283-292.

Rodriguez, P. C., Ochoa, A. C., \& Al-Khami, A. A. (2017). Arginine metabolism in myeloid cells shapes innate and adaptive immunity. Frontiers in Immunology, 8,93 .

Schreiner, B., Mitsdoerffer, M., Kieseier, B. C., Chen, L., Hartung, H. P., Weller, M., \& Wiendl, H. (2004). Interferon-beta enhances monocyte and dendritic cell expression of B7-H1 (PD-L1), a strong inhibitor of autologous T-cell activation: Relevance for the immune modulatory effect in multiple sclerosis. Journal of Neuroimmunology, 155, 172-182.

Shin, W., Berkowitz, D. E., \& Ryoo, S. (2012). Increased arginase II activity contributes to endothelial dysfunction through endothelial nitric oxide synthase uncoupling in aged mice. Expimental and Molecular Medicine, 44, 594-602.

Sica, A., Erreni, M., Allavena, P., \& Porta, C. (2015). Macrophage polarization in pathology. Cellular and Molecular Life Sciences, 72(21), 4111-4126.

Thomas, A. C., \& Mattila, J. T. (2014). "Of mice and men": Arginine metabolism in macrophages. Frontiers in Immunology, 5, 479.

Tupotilov, O. V., \& Kolyada, T. I. (2018). Citokinogenez pri TLR-oposeredkovanyj aktyvaciji monocytiv peryferychnoji krovi u hvoryh z rozsijanym sklerozom [Cytokinogenesis by TLR-induced activation of peripheral blood monocytes in patients with multiple sclerosis]. Visnyk Problem Biologiji i Medycyny, 145, 181-185 (in Ukrainian).

Vdovichenko, N., Koliada, O., \& Tupotilov, O. (2020). Osoblyvosti tsytokinovoho profiliu v patsyentiv z riznymy typamy perebihu rozsijanoho sklerozu [Features of cytokine profile in patients with different types of multiple sclerosis]. Immunology and Allergology: Science and Practice, 1, 12-16 (in Ukrainian).

Wiesemann, E., Deb, M., Trebst, C., Hemmer, B., Stangel, M., \& Windhagen, A. (2008). Effects of interferon-beta on co-signaling molecules: upregulation of CD40, CD86 and PD-L2 on monocytes in relation to clinical response to interferon-beta treatment in patients with multiple sclerosis. Multiple Sclerosis, 14(2), 166-176.

Xie, Z. X., Zhang, H. L., Wu, X. J., Zhu, J., Ma, D. H., \& Jin, T. (2015). Role of the immunogenic and tolerogenic subsets of dendritic cells in multiple sclerosis. Mediators of Inflammation, 2015, 513295. 